\title{
Gross Tumour of Minor Salivary Glands in HIV Sero-Positive Patient
}

\author{
Jean Valentin Fokouo Fogha, Gisele Alenda Ngulefac, Esthelle Genevieve Stephanie Minka Ngom, \\ Vincent Fonyam, Louis Richard Njock
}

Douala General Hospital, Douala, Cameroon.

Email: ngualenda@yahoo.com

Received January $8^{\text {th }}, 2012$; revised February $10^{\text {th }}, 2012$; accepted March $14^{\text {th }}, 2012$

\begin{abstract}
Tumours of minor salivary glands are rare. We report the case of a 58-year-old HIV positive woman with a palatal adenocarcinoma evolving for 15 years, followed by a literature review. The case enlightens on the deleterious effect of delayed specialist consultation, which in our context is multifactorial in origin.
\end{abstract}

Keywords: Adenocarcinoma; Minor Salivary Glands; HIV

\section{Introduction}

Tumours of minor salivary glands are rare [1]. The palate is the most frequent site of affection. We report the case of an HIV sero-positive patient who presented with a voluminous tumour of the palate.

\section{Clinical Case}

A 58 year-old female, farmer, known HIV sero-positive since 6 years and on antiretrovirals, who inhales tobacco, was referred to our service in December 2010 for a tumour of the buccal cavity evolving since 15 years. The clinical presentation was characterised by aggravating dysphagia. The tumour had been operated in a non-specialised service without histopathology examination and evolution was characterised by a temporary amelioration. Since 2006, there was reinstallation of aggravating dysphagia with weight loss, in addition to bilateral nasal obstruction, persistent rhinorrhoea and hyponasality.

Physical examination revealed weight loss of about $30 \%$ of initial body weight and buccal respiration. Figure 1 below shows facies of the patient with open mouth breathing. In the buccal cavity was a voluminous tumour of the palate which extended from the hard palate to the base of the tongue, obstructing the isthmus of Gosier as shown on Figure 2. The surface which was smooth and regular was lined by blood vessels and there was a small ulceration. The mass was firm and non tender. Rhinoscopy revealed total obstruction of both nasal fossae. Buccal opening was normal and the lymph node areas were free.

A CT scan of the facial complex showed a mass of the soft palate with lysis of the palatal roof (Figure 3) and the maxillary sinus, and extending to the sphenoid, nasal

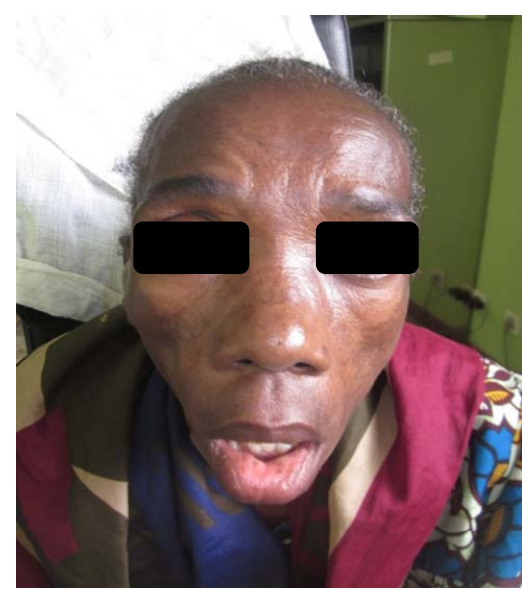

Figure 1. Facies with buccal respiration.

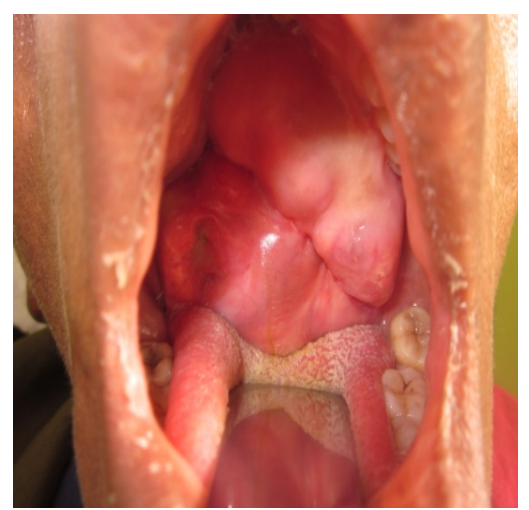

Figure 2. Endobuccal view. 

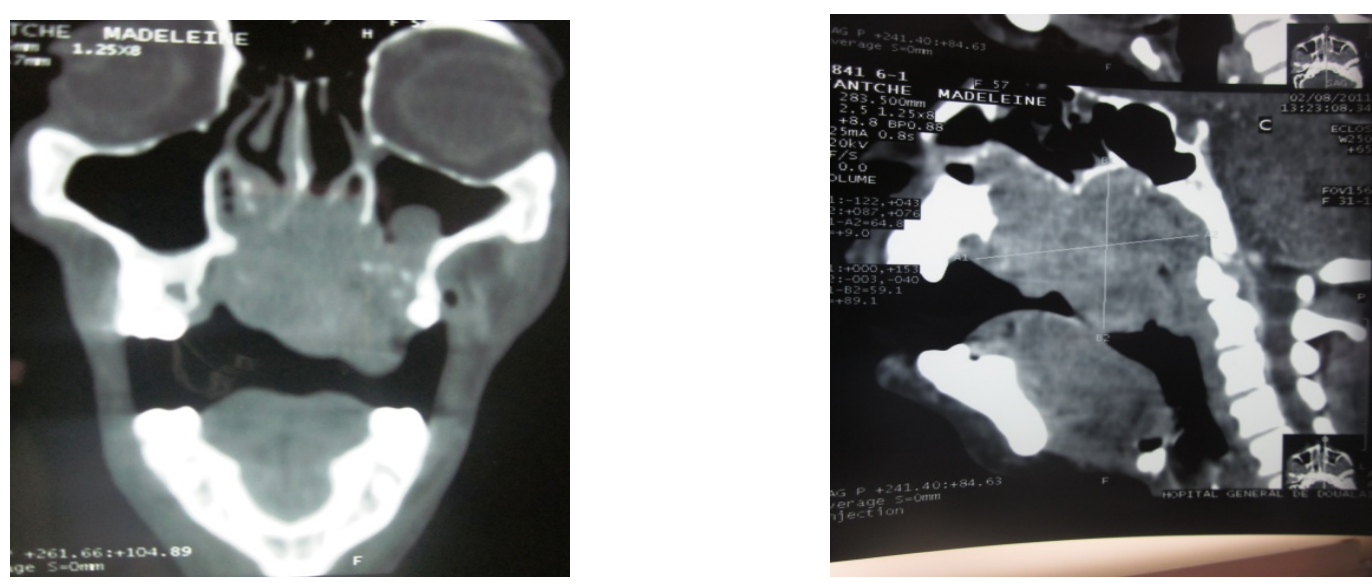

Figure 3. Coronal and sagittal cuts showing tumour extension and lysis of palatine, maxilla and sphenoid bones.

fossae and infratemporal fossa, without intracranial extension.

Biopsies and histopathologic examination revealed a well differentiated adenocarcinoma of the accessory salivary glands. Extensive workup showed no pulmonary or hepatic metastasis.

A multidisciplinary therapeutic decision-making meeting with the Otolaryngologist, the medical oncologist and the radiation therapist concluded that the patient was not eligible for radiation therapy or chemotherapy due to her general state. Surgery on the tumour not feasible due to the locoregional extension, a gastrostomy was proposed for feeding as well a tracheotomy.

\section{Discussion}

Tumours of salivary glands are rare, representing about $3 \%$ of tumours of the head and neck [1,2]. According to Boko, tumours of salivary glands represent $17.19 \%$ of ENT tumours in Togo [2]. Those of minor salivary glands represent 10 to $15 \%$ of tumours of salivary glands in the Janisyanont series [3]. Like in our clinical case, females are the most affected, with sex ratio of 1.2 to 1.9 female to male [3]. The age of our patient is comparable to the mean age of development of these tumours by Boko [2], which was 38 years if we consider the duration of evolution of her tumour, about 15 years.

The delay before ENT consultation with administration of inappropriate treatment in non-specialised context retarded the diagnosis and adequate management. This is a frequent problem in practice due inaccessibility of main hospital centres to patients and the small number of available specialists. This pushes the general physician to accomplish many tasks reserved for the specialist. In our case, the macroscopic aspect of the tumour apart from the recent ulceration was not suspicious of a malignancy. Jansisyanont underlines the impossibility to differentiate clinically a benign from a malignant tumour [3]. Apart from the enormous size of the tumour, the clinical presentation was classical.

The tumour of our patient was a well differentiated adenocarcinoma. In the different series in literature, reports are variable, with predominance of malignant forms on one hand [1,3] and benign forms on the other hand [2]. The most frequent malignant form by studies is the cystic adenoid carcinoma [1,2] or the mucoepidermoid carcinoma [3]. The predominance of benign forms in African studies is explained by Isacsson and Boko [2,4] by frequency of pleomorphic adenomas which is 3.5 times more frequent in blacks than Caucasians [4].

The HIV sero-positive state of our patient seems to be a circumstantial association. Actually, literature does not give any relation between HIV and tumours of minor salivary glands.

With the extensiveness of the tumour and lysis of the left pterygoid process, we would have expected to have trismus in this patient but for an unknown reason, it was absent.

The treatment was palliative. If otherwise diagnosed earlier, surgical resection only could have been sufficient.

\section{Conclusion}

Adenocarcinoma of minor salivary glands remains rare as for all tumours of the accessory salivary glands. Late consultation is the usual scenario as for most cancers of the upper aero-digestive tract. Late referral to a specialised centre aggravates prognosis.

\section{REFERENCES}

[1] P. Bonfils, “Tumeurs des Glandes Salivaires, in Encyclopédie Médico-Chirurgicale ORL,” Elsevier, Paris, 2004,.

[2] E. Boko, et al., "Tumours of the Accessory Salivary Glands. Epidemiological and Anatomopathological Aspects," Revue Laryngologie Otologie Rhinologie, Vol. 125, No. 4, 2004, pp. 233-237. 
[3] P. Jansisyanont, R. H. Blanchaert Jr. and R. A. Ord, "Intraoral Minor Salivary Gland Neoplasm: A Single Institution Experience of 80 Cases," International Journal of Oral Maxillofacical Surgery, Vol. 31, No. 3, 2002, pp. 257-261. doi:10.1054/ijom.2002.0223
[4] G. Isacsson and M. Shear, "Intraoral Salivary Gland Tumors: A Retrospective Study of 201 Cases,” Journal of Oral Pathology \& Medicine, Vol. 12, No. 1, 1983, pp. 5762. doi:10.1111/j.1600-0714.1983.tb00316.x 